\title{
Simple noninterference mechanism between the pitch and yaw axes for a wrist mechanism to be employed in robot-assisted laparoscopic surgery
}

\author{
Makoto Jinno* (i)
}

\begin{abstract}
Laparoscopic surgery, which is also called minimally invasive surgery, is a surgical technique that is associated with accelerated post-operative recovery. However, it can only be performed by surgeons possessing advanced surgical skills. One of the main challenges in laparoscopic surgery is the restriction of the free motion of forceps because of the limited degrees of freedom imposed by the trocar. Recently, to overcome this problem, many master-slave manipulators with articulated forceps have been used in laparoscopic surgery. The wrist mechanism of the articulated forceps affects the controllability and range of motion of the slave manipulator in the abdominal cavity of a patient. Therefore, improvement of the wrist mechanism of the articulated forceps is important for robot-assisted laparoscopic surgery. This study proposes a new wrist mechanism for using articulated forceps in laparoscopic surgery. The degrees of freedom of the proposed design are provided by motor-driven or manually driven axes employing various wires and pulleys (pitch, yaw, and gripper axes). The kinematic model of this mechanism is decoupled between the pitch axis and yaw axis by a very simple mechanism using arc-shaped guides and wire guide holes. The arc-shaped guides minimize the wire path length error of the yaw and gripper axis wire resulting from the motion of the pitch axis. The optimized position of the arc-shaped guides is decided by the minimal root-mean-square value of the wire path length error. The proposed wrist mechanism has only half the number of parts as compared to the previously developed robotic forceps for clinical use. Furthermore, the effectiveness of the proposed mechanism was demonstrated on a prototype model with a maximum outer diameter of $7.5 \mathrm{~mm}$. Conversely, the disadvantages of the proposed mechanism lie in the transmission mechanism efficiency and no-load input torque.
\end{abstract}

Keywords: Minimally invasive surgery, Manipulator, Wire drive mechanism, Noninterference mechanism

\section{Background}

Laparoscopic surgery is a commonly employed minimally invasive surgical techniques. In the case of conventional laparoscopic surgery, a surgeon operates using forceps passed through trocars (each approximately $5-10 \mathrm{~mm}$ in diameter) into a patient's abdominal cavity while observing the image acquired by a laparoscope. Since only a small incision is made, the patient can recover sooner,

*Correspondence: mjinno@kokushikan.ac.jp

School of Science and Engineering, Kokushikan University, 4-28-1, Setagaya, Setagaya-ku, Tokyo 154-8515, Japan and the overall medical costs are reduced. Therefore, laparoscopic surgery has great advantages for the patient, and its use has become widespread in recent years. However, laparoscopic surgery can only be performed by surgeons with advanced skills. One of the main difficulties in laparoscopic surgery is the restriction of the free motion of the forceps because of the size of the trocar. The position and posture of the gripper of the forceps cannot be changed freely in the patient's abdominal cavity during the procedure because the shaft of the forceps is restricted by the trocar. Recently, many master-slave manipulators with articulated forceps have been used in 
laparoscopic surgery to solve this problem [1]. Compact and low-cost manually driven or motor-driven handheld articulated forceps have been developed for clinical use [2-4].

The wrist mechanism of an articulated forceps affects the controllability and range of motion of a slave manipulator or handheld articulated forceps in the abdominal cavity of a patient. Therefore, it is very important to improve upon the wrist mechanism of articulated forceps for robot-assisted laparoscopic surgery. This study focused on noninterference wrist mechanisms. The most popular wrist mechanism, the da Vinci [1] mechanism, adopts a wire-pulley drive mechanism [5]. However, this is an interference mechanism. Furthermore, the wrist mechanisms reported in previous papers $[6,7]$ and a patent [8] proposed by the author are interference mechanisms. In general, articulated forceps that employ a wire-pulley or gear drive mechanisms or both are interference mechanisms. The demerits of this mechanism are discussed in the next section.

On the other hand, several noninterference wrist mechanisms for articulated forceps have been proposed. The wrist mechanism of the Kymerax [4], a system of handheld articulated robotic forceps, adopts a combination of the wire-pulley drive and gear drive mechanism [9]. The mechanism of the gripper axis is decoupled from the pitch and roll axis. Thus, a hybrid drive mechanism of the manual drive gripper axis and motor drive pitch and yaw axes is realized. Nishizawa and Kishi [10] proposed a noninterference mechanism using a wire-pulley and gear mechanism. In the mechanism, a middle plate connects two parts that make rolling contact with each other. Therefore, the path length of the wires is independent of the joint angle. The wrist mechanism of the Radius [3] is a combination of the torque tube, gear, and link drive mechanisms [11]. The mechanism of the gripper axis is decoupled from pitch and roll axes. However, it is not suitable for dissections since the open motion of the gripper is driven by a spring force and the dissecting force is small. Compared to interference mechanisms, the noninterference mechanisms need more parts to realize decoupling between joints.

Ikuta et al. [12] proposed a noninterference mechanism for a remote microsurgery system to use in deep and narrow spaces. In the mechanism, the driving wire of the tip joint passes through the center of the base joint, the length of the wire path remains constant regardless of the angle at which the base joint is bent. However, as the wire is bent in a small radius, smooth motion and durability may be problematic.

The FlexDex [2] uses a flexible bending joint and wire drive mechanism [13]. Furthermore, Lee and Chamorro adopted a flexible bending joint and wire drive mechanism [14]. Hraguchi et al. [15] also proposed a flexible bending joint and wire drive mechanism using a spring for the joint. Bending mechanisms using flexible joints are very simple. The mechanisms of the flexible bending joint and wire are decoupled between the axes. Therefore, many handheld robotic forceps adopt this mechanism. However, since the bending radius of the flexible joint is large with respect to the forceps diameter, large motion is required to determine the posture of the end effector. It is difficult to perform procedures in a narrow space when using forceps with a flexible bending joint.

Considering the need for good controllability and a simple wrist mechanism for robot-assisted laparoscopic forceps, this study explores an extremely simple wrist mechanism using the wire drive mechanism. In this paper, first, the demerits of interference wrist mechanism and conventional noninterference mechanisms are examined. Next, a new noninterference wrist mechanism with arc-shaped guides and wire guide holes is proposed and the design of the optimized arc-shaped guide position is explored. Third, a prototype model of the wrist mechanism is described. Finally, the experimental results obtained using a test bench to validate the proposed mechanism are reported.

\section{Methods}

This section examines the demerits of the interference wrist mechanisms and conventional noninterference mechanisms. A new noninterference mechanism between the pitch and yaw axes and the optimum design method for the arc-shaped guide that can overcome the aforementioned disadvantages are proposed.

\section{Demerits of interference wrist mechanisms}

When designing articulated forceps for laparoscopic surgery, it is very difficult to dispose the actuators at the distal end of the forceps to miniaturize and thinning. Hence, they are located on the proximal end of the forceps shaft. Therefore, the power of the actuators located outside the abdominal cavity of a patient is transmitted to the distal end of the forceps using power transmission mechanisms such as wires, pulleys, links, rods, torque tubes, or gears. Mechanical interference occurs commonly because the power transmission mechanisms are disposed in the joints and since the power is passed through the joints. By controlling the angle of the actuator based on the mechanism interference matrix, it is possible to control the joint angle (yaw axis and pitch axis) and gripper angle (gripper axis) of the distal end portion of the forceps. However, the following problems are encountered.

1. Increase in the motion range of the actuators. 
2. Increase in the actuator torques caused by interference torque from other joints.

3. Uncontrollable joint motion caused by interference torque from other joints. For example, when the yaw joint moves, the pitch joint is slightly shifted by the interference torque from the yaw joint. This can be confirmed as shown in "Additional file 1. Demonstration motion of each axis by a tactile-switch user interface" in Ref. [7].

In particular, uncontrollable joint motion is the most serious issue. This problem can be solved by the following methods.

1. Increase the joints stiffness, including servo stiffness, by using wires with high tensile stiffness to reduce elongation or using a large-diameter pulley or by both these methods; Especially, the joint stiffness is proportional to the square of the pulley radius.

2. Interference torque compensation; for example, modify the desired position of the pitch axes to compensate for interference torque simultaneously with yaw axis operation [16].

3. Incorporation of the noninterference mechanism as a fundamental solution to avoid uncontrollable joint motion.

\section{Conventional noninterference mechanism}

Several noninterference wrist mechanisms for articulated forceps have been proposed. In a noninterference wrist mechanism, the mechanism should not change the path length of power transmission, such as wire routing length, and the intermeshing point of the gear accompanying the motion of other axes. Two types of noninterference wrist mechanisms were proposed.
1. For two curvature axes [13-15].

In the case of two curvature axes for up/down and right/left motions using a flexible bending joint and wire drive mechanism, the up/down and right/left bending motions are decoupled from each other because the wires are routed on a plane passing through the bending center of the other axes. Furthermore, the power transmission parts of the gripper axis are routed along the central axis of the forceps shaft. Then, the gripper axis is decoupled from the up/down and right/left axes. The flexible bending joint and wire drive mechanisms are very simple. However, since the bending radius of the flexible joint is larger than the forceps diameter, large motion is required to determine the posture of the end effector. Performing procedures in a narrow space with a forceps with a flexible bending joint is difficult.

2. For a combination of bending and/or roll axes $[9,10]$. When two bending axes (a pitch axis orthogonal to the shaft axis and a yaw axis, or vice versa) or a bending axis (pitch axis or yaw) and a roll axis are used, the bending and rolling motions are decoupled from each other using power transmission parts such as wires, pulleys, links, and gears. However, compared to the interference mechanisms, more parts are required in the noninterference mechanisms.

\section{Proposed noninterference mechanism configuration}

Figure 1 shows the conceptual design of a wrist mechanism of articulated forceps with the proposed noninterference mechanism between the pitch and yaw axes. It also shows the shape of the supporting part that plays an important role in this mechanism. The wrist mechanism consists of a supporting part, a pitch part, and a part each for the right finger and left finger for an end effector,

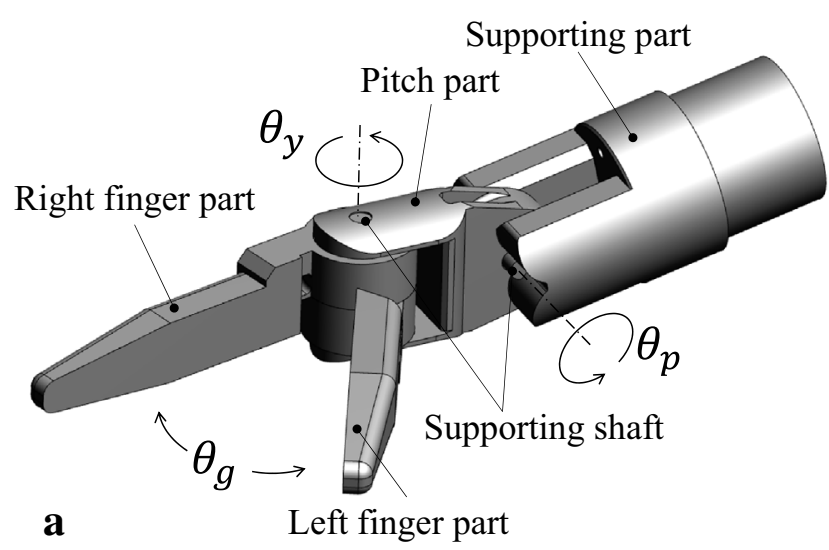

Wire-guide hole

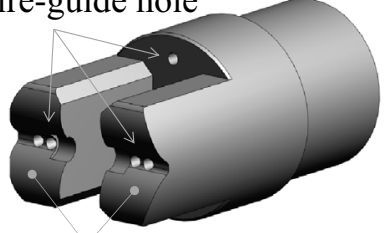

Arc-shaped guide

b

Fig. 1 Conceptual design of the proposed wrist mechanism. a Overview; b supporting part 
supporting shafts, and stainless wires (not shown). The shape of the pitch part is roughly the same as that employed in a previous work [7].

If the wire path that bends at the center of the pitch axis can be constructed, the path length of the 2nd-axis and 3rd-axis wires accompanying the pitch axis motion does not change. However, considering the durability of the wire and smooth wire operation, the bending wire path at the center is not adopted because it is necessary to maintain the radius. On the other hand, in the proposed wrist mechanism, arc-shaped guides are located on the upper and lower portions of the plane, including the pitch axis and the central axis of the supporting part. The arc-shaped guide provides sufficient bending radius of the wire and minimizes the change in the path length of the 2nd-axis and 3rd-axis wires arising from pitch axis motion. Compared with the case of using pulleys, there is concern about friction between the wire and circular arc-shaped surface, but the bending radius of the wire can be greatly increased and the number of parts can be greatly reduced.

The supporting part has two through-holes for the wire to drive the pitch part located at the upper and lower portions of the supporting part. In addition, it has four through-holes for the 2nd- and 3rd-axis wires to drive the right and left finger parts for realizing yaw and grasping motions. Four through-holes are located in parallel with the pitch axis on the plane, including the pitch axis and the central axis of the supporting part. Figure 2 shows the path of the wire. The winding angle of the wire to the pulley is $540^{\circ}$ ( 1.5 turns), but in the figure, this angle is shown to be $180^{\circ}$ (half turn) to simplify the drawing for easy understanding.

\section{Design of the optimized arc-shaped guides position (for avoiding bending of the wire at the arc-shaped guide end)}

This section describes the design method to optimize position of the arc-shaped guides. Figure 3 shows examples of the 2nd-axis and 3rd-axis wire positions when the wire pitch angle $\theta_{P}=0$ and $90^{\circ}$. When $\theta_{P}=0^{\circ}$, the 2ndaxis and 3rd-axis wires stay straight after passing through the holes; on the other hand, when $\theta_{P}=90^{\circ}$, they follow the arc-shaped guide. Figure 4 shows the relationship

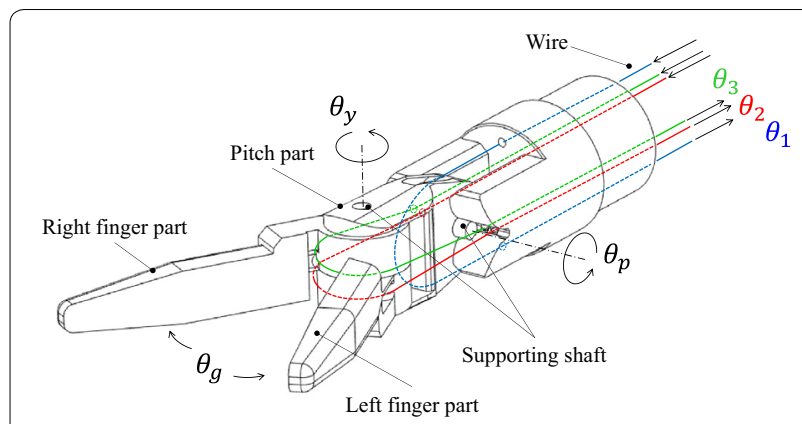

Fig. 2 Wire path of the proposed wrist mechanism

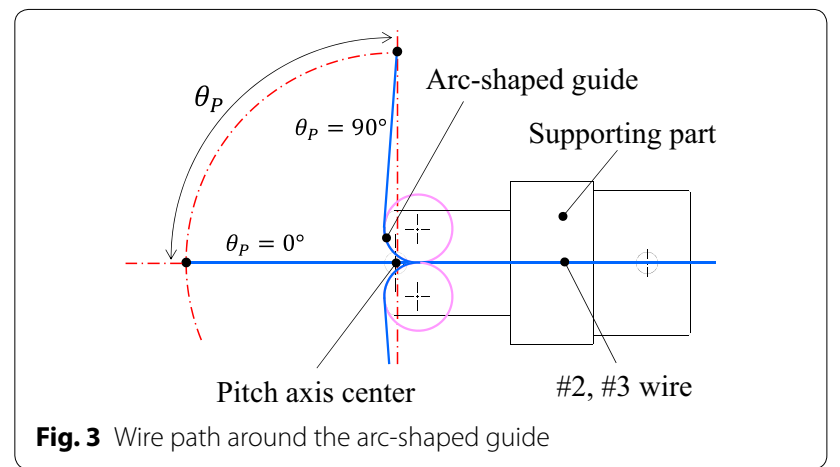

between the arc-shaped guide radius $r_{1}$, the arc-shaped guide center $\left(x_{a}, y_{a}\right)$, the rotation radius of wire tip around pitch axis $r_{2}$, etc. When designing the supporting part, the radius of the arc-shaped guide must be $r_{1}-D / 2$, considering the wire diameter $\phi D$.

To avoid bending the wire at the arc-shaped guide end, it is preferable to set the arc-shaped guide tangential to the x-axis. In other words, set $y_{a}=r_{1}$. When $\theta_{P}=0^{\circ}$, the wire length $l$ is $l=r_{2}+x_{a}$, and when $\theta_{P}>0^{\circ}$, the wire length $l$ is $l=l_{a}+l_{b}$. Here $l_{b}$ is the wire length on the arcshaped guide. Therefore, since the ideal state in which the wire path length does not change is $r_{2}+x_{a}=l_{a}+l_{b}$, the wire path length error is defined as follows.

$$
e=\left(l_{a}+l_{b}\right)-\left(r_{2}+x_{a}\right) .
$$

The wire path length $l=l_{a}+l_{b}$ can be obtained from the analytical solution geometrically by considering the flow, as shown in Fig. 5. Figure 6 shows the relationship between angle $\theta_{P}$ and wire path length error $e$, when the arc-shaped guide center position $x_{a}$ is varied from 0 to $1.6 \mathrm{~mm}$ for the following parameters: $r_{1}=2 \mathrm{~mm}$, $y_{b}=2 \mathrm{~mm}$, and $r_{2}=9.2 \mathrm{~mm}$. As shown in Fig. 6, the wire path length error $e$ varies depending on pitch angle $\theta_{P}$ and the arc-shaped guide position $x_{a}$. Figure 7 shows the relationship between the arc-shaped guide center position $x_{a}$ and the root-mean-square (RMS) of the wire path length error $e$. As shown in Fig. 7, when the motion range of the pitch angle $\theta_{P}$ varies from $0^{\circ}$ to $90^{\circ}$, the RMS of the wire path length error $e$ becomes minimum for an arc-shaped guide center position $x_{a}=1 \mathrm{~mm}$. The optimized position of the arc-shaped guides can be decided from the minimal RMS of the wire path length error. In the case of $r_{1}=1.5 \mathrm{~mm}, 1.75 \mathrm{~mm}, 2.25 \mathrm{~mm}, 2.5 \mathrm{~mm}$, each center position of the arc-shaped guide of minimal RMS of the wire path length error is $x_{a}=0.75 \mathrm{~mm}, 0.875 \mathrm{~mm}, 1.125 \mathrm{~mm}, 1.25 \mathrm{~mm}$, respectively. The same results were obtained even when the arc-shaped guide radius changed. Therefore, the optimized position of the arc-shaped guides is obtained by an approximate expression shown by the following equation. 


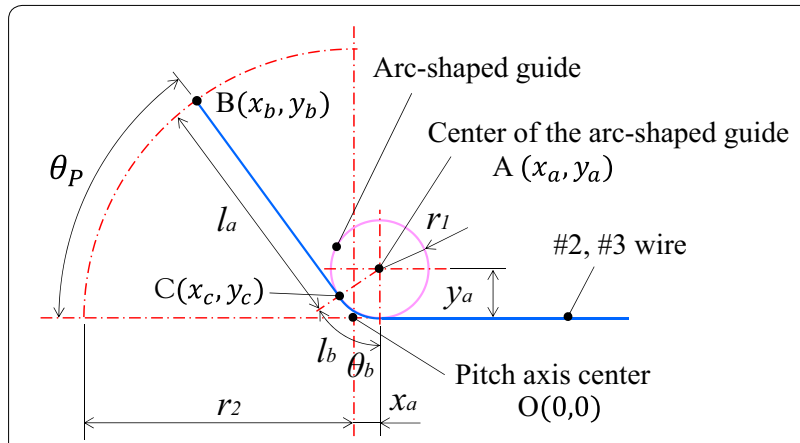

Fig. 4 Wire path length calculation model
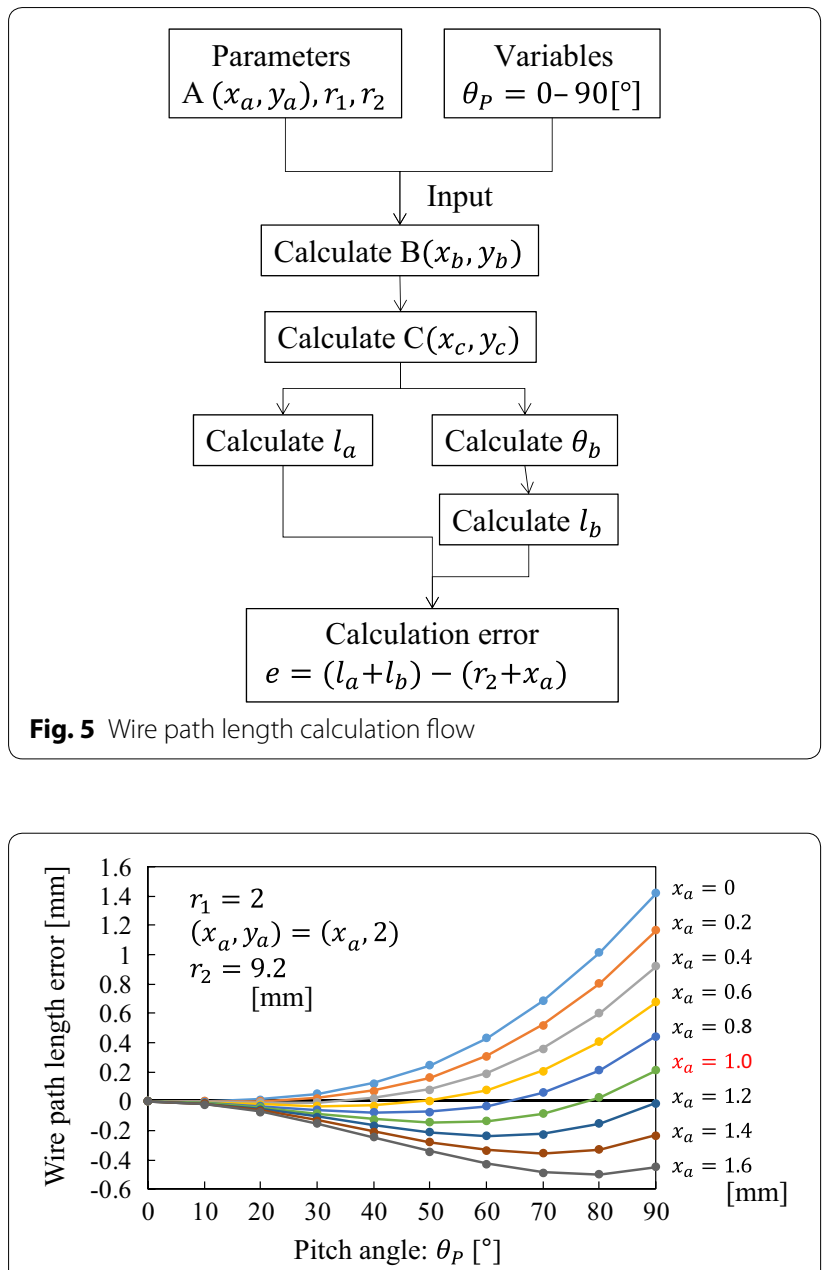

Fig. 6 Wire path length error

$$
\left(x_{a}, y_{b}\right)=\left(\left(\frac{r_{1}}{2}\right), r_{1}\right) .
$$

Similarly, when the motion range of the pitch angle $\theta_{P}$ is $0^{\circ}$ to $n^{\circ}$, the optimized arc-shaped guide center positions can be obtained by an approximate expression shown by the following equation.

$$
\left(x_{a}, y_{b}\right)=\left(\left(\frac{r_{1}}{2}\right)\left(\frac{n}{90}\right), r_{1}\right) .
$$

\section{Design of the optimized arc-shaped guides position (for obtuse angle bending of the wire at the arc-shaped guide end)}

Since the wire is flexible, when there is obtuse angle bending of the wire at the arc-shape guide end, a reduction in the wire path length error can be expected. As shown in Fig. 8, in the case in which wire bending is allowed at the arc-shaped guide end, the position of the arc guide is modified by the following steps.

1. Move the center of the arc-shaped guide slightly toward the positive $\mathrm{x}$-axis and negative $\mathrm{y}$-axis.

2. Cut out the $y<0$ part of the guide.

The maximum allowable bending angle of the wire was set at approximately $30^{\circ}$ during this evaluation. However, ultimately, it is necessary to decide whether to adopt it after considering the durability of the wire. For designing articulated forceps with a shaft diameter of $\phi 7.5 \mathrm{~mm}$, the arc-shaped guide radius $r_{1}$ should be $1.75 \mathrm{~mm}$, including the wire diameter, and the rotation radius of the wire tip around the pitch axis $r_{2}$ should be $9.7 \mathrm{~mm}$. When the bending of the wire is avoided, the RMS of the wire path length error obtained using Eq. (1) is minimum at $P_{A}\left(x_{a}, y_{a}\right)=(0.875 \mathrm{~mm}, 1.75 \mathrm{~mm})$. On the other hand, when allowing bending angle of the wire, following result was obtained by trial and error. The wire path length error tends to decrease in the region where $x_{a}>0.875 \mathrm{~mm}$ and $y_{b}<1.75 \mathrm{~mm}$. Therefore, the wire path length error $e$ and the RMS and the maximum wire bending angle for the vicinity of the arc-shape guide center position $P_{A}=(1.0 \mathrm{~mm}, 1.55 \mathrm{~mm})$ were examined.

Figure 9 shows the path length error for the vicinity of the arc-shaped guide center position $P_{A}=(1.0 \mathrm{~mm}, 1.55 \mathrm{~mm})$ for (a) comparison along the $y$-axis and (b) comparison along the $x$-axis. Figure 10 shows the RMS of the wire path length error for Fig. 9a, b. Figure 11 shows the maximum wire bending angle at the arc-shaped guide end. The optimum position along the $\mathrm{y}$-axis is $y_{b}=1.55 \mathrm{~mm}$ from Figs. 9a and $10 \mathrm{a}$, and the optimum position in the $\mathrm{x}$-axis direction is $x_{a}=1.0 \mathrm{~mm}$ from Figs. $9 \mathrm{~b}$ and $10 \mathrm{~b}$. The reason is as follows. In Fig. 10a, the RMS of the wire path length error with $\theta_{P}=0-90^{\circ}$ is slightly smaller for $y_{b}=1.6 \mathrm{~mm}$ than for $y_{b}=1.55 \mathrm{~mm}$. Furthermore, in Fig. 10b, the RMS of the wire path length error for $\theta_{P}=0-90^{\circ}$ is slightly smaller for $x_{a}=1.1 \mathrm{~mm}$ than for $x_{a}=1.0 \mathrm{~mm}$. However, 


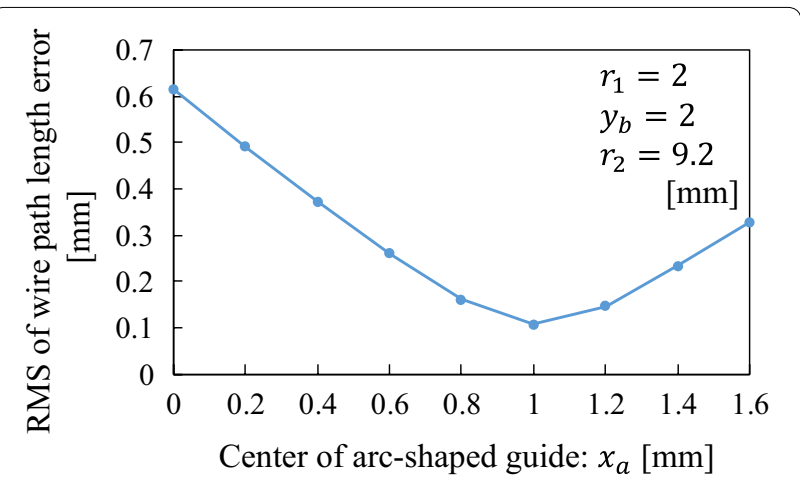

Fig. 7 Root-mean-square (RMS) of wire path length error considering that the center of the main operating range is $\theta_{P}=0^{\circ}$, the motion range where $\theta_{P}$ is small is very important. Therefore, the optimum is $P_{A}=(1.0 \mathrm{~mm}, 1.55 \mathrm{~mm})$. Furthermore, the maximum wire bending angle at the arc-shape guide end is $27.7^{\circ}$. This value is smaller than the allowable bending angle. Furthermore, the negative sign of the wire path length error means that the wire path length decreases. Since the wire is pre-tensioned, the wire path length tends to become shorter. Therefore, reducing the wire path length error as small as much as possible in the vicinity of $\theta_{P}=0^{\circ}$, which is the center of the main operating range, helps realize smooth operation with the 1st axis (pitch axis). Figure 12 compares the path length error for the optimized position $P_{A}=(1.0 \mathrm{~mm}, 1.55 \mathrm{~mm})$ and the position to avoid

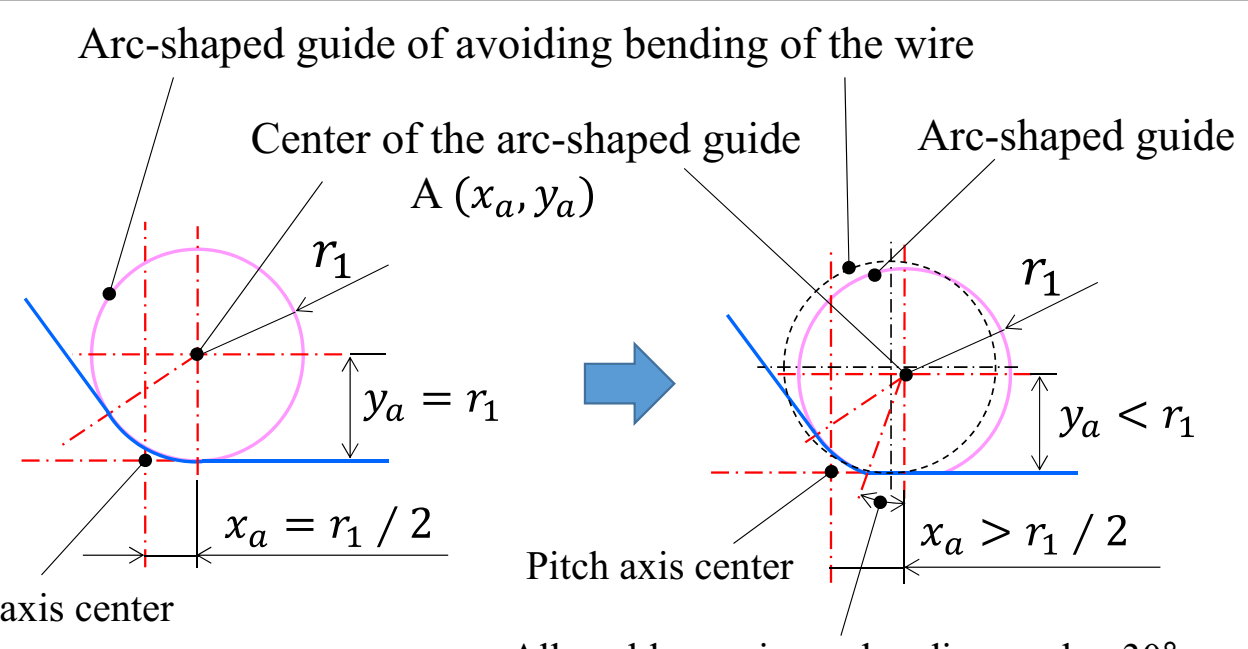

Allowable maximum bending angle : $30^{\circ}$

Fig. 8 Allowable maximum bending angle at the arc-shape guide end
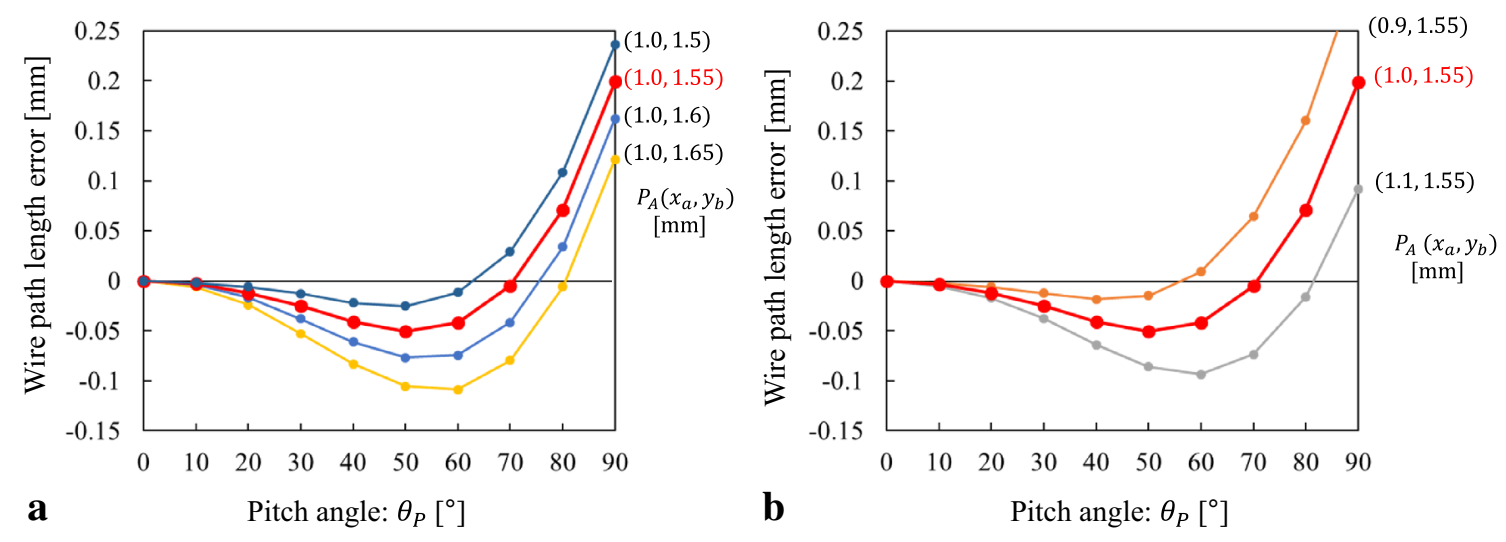

Fig. 9 Wire path length error for the vicinity of optimized position. $\mathbf{a}$ Comparison in the $y$-axis direction; $\mathbf{b}$ comparison in the $x$-axis direction 


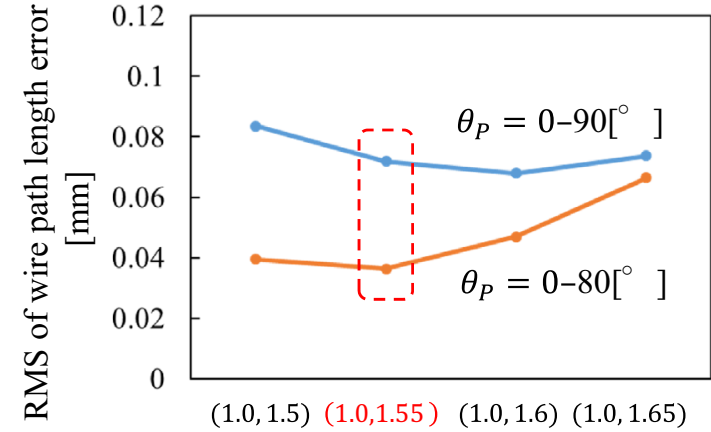

a

Center of arc-shaped guide : $\left(x_{a}, y_{a}\right)[\mathrm{mm}]$

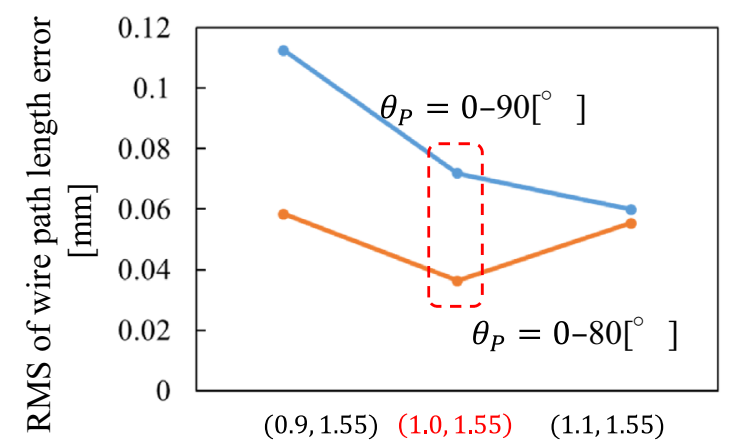

Center of arc-shaped guide : $\left(x_{a}, y_{a}\right)[\mathrm{mm}]$

Fig. 10 Root-mean-square (RMS) of wire path length error in the vicinity of the optimized position. a Comparison in the $y$-axis direction; $\mathbf{b}$ comparison in the $\mathrm{x}$-axis direction

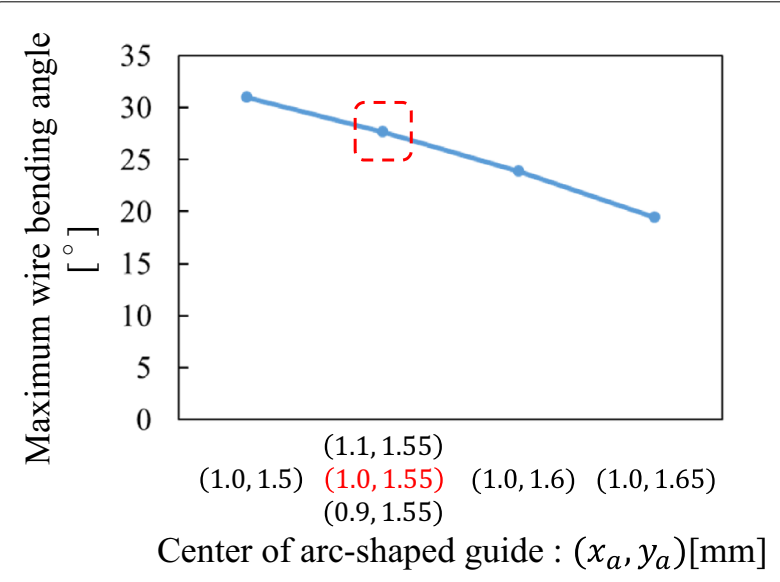

Fig. 11 Maximum wire bending angle at the arc-shaped guide end

bending of the wire $P_{A}=(0.875 \mathrm{~mm}, 1.75 \mathrm{~mm})$. The wire path length error of $\theta_{P}=0-70^{\circ}$ is an extremely small value compared to the error of the position for avoiding bending of the wire. In addition, in the RMSs of the wire path length errors of $\theta_{P}=0-90^{\circ}$ and $0-80^{\circ}$ improved to 0.074 and $0.036 \mathrm{~mm}$ from 0.092 and $0.071 \mathrm{~mm}$, respectiv ely.

\section{Specification of the wrist mechanism}

Figure 13 shows the mechanical design of the proposed noninterfering wrist mechanism, and Table 1 lists the specifications. The maximum outer diameter is $7.5 \mathrm{~mm}$, which is equivalent to that of the conventional wrist mechanisms used in articulated forceps for robot-assisted laparoscopic surgery [5, 7]. The offset distance between the pitch axis and the yaw axis is $6.95 \mathrm{~mm}$. The radius of the arc-shaped guide of the supporting part is $1.5 \mathrm{~mm}$. The wire diameter is $0.45 \mathrm{~mm}$. Therefore, $r_{1}=1.5+0.45 / 2 \mathrm{~mm}$. In addition, the

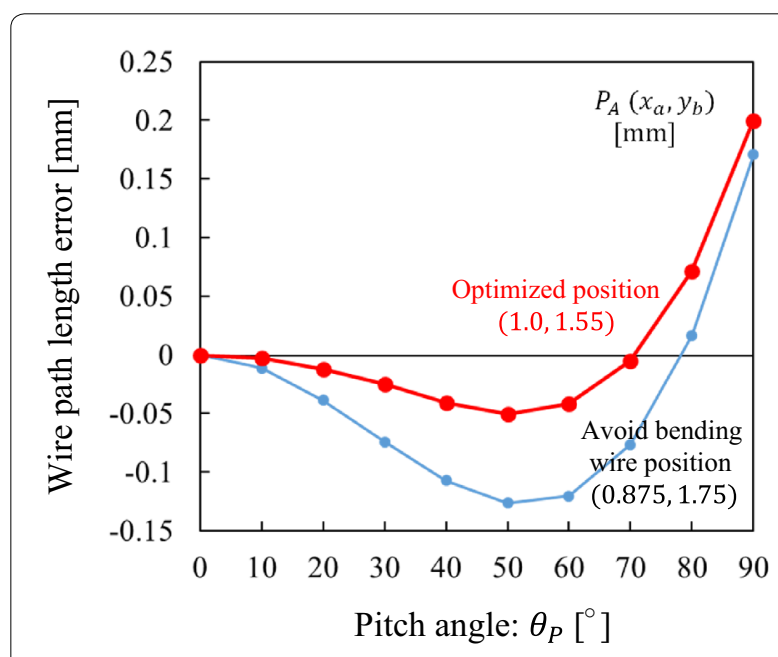

Fig. 12 Comparison of the wire path length error between the optimized position and the position for avoiding the bending of the wire

rotation radius of the wire tip around the pitch axis is $r_{2}=9.45+0.45 / 2 \mathrm{~mm}$. Table 2 presents a comparison of the components with the parts of the conventional wrist mechanism [7]. Figure 14 shows the wire route and coordinate system. Table 3 lists the pulley diameter and the wire pitch diameter values.

The wrist mechanism consists of the minimum necessary parts that are indispensable. The great reduction in the number of parts and the simplification is expected to reduce the expenses, such as the manufacturing, management, and assembly costs for the parts and to improve the cleaning and sterilization ability. Furthermore, it is possible to use this mechanism in conjunction with hand-held robotic forceps to apply a grasping force that can be directly and manually controlled by surgeons. It is 


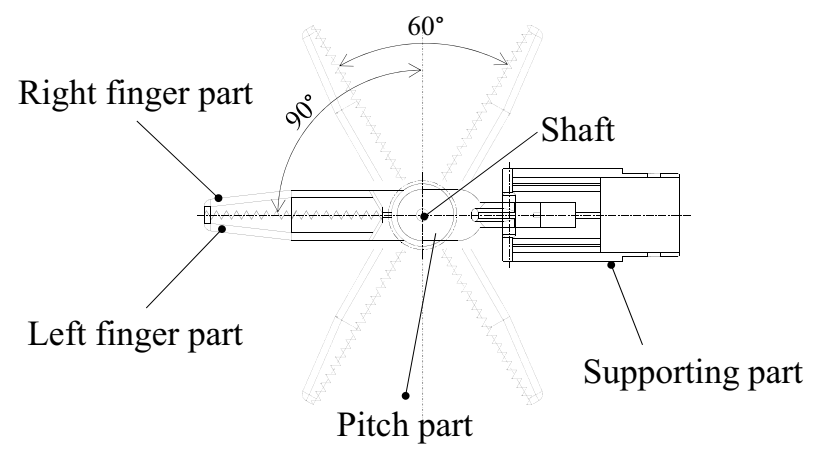

a

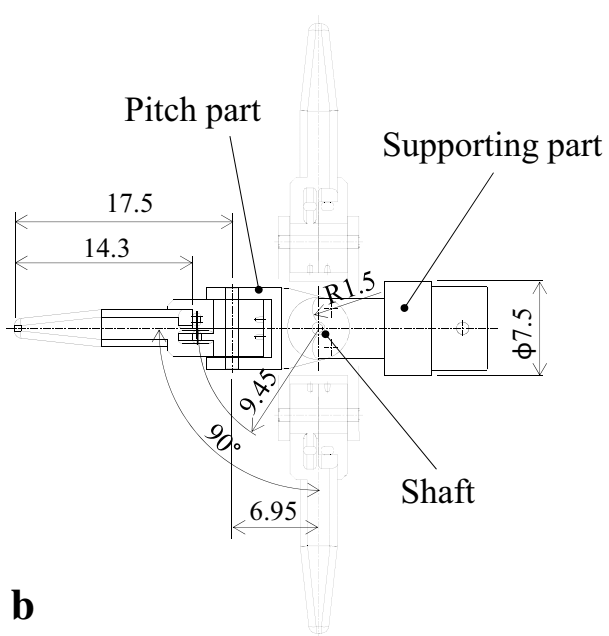

Fig. 13 Design of wrist mechanism. a Yaw- and gripper-axis motion range (top view); b pitch axis motion range (front view)

Table 1 Specifications of the wrist mechanism

\begin{tabular}{ll}
\hline Items & Specification \\
\hline Size & \\
Maximum diameter & $\phi 7.5 \mathrm{~mm}$ \\
Offset from pitch axis to yaw axis & $6.95 \mathrm{~mm}$ \\
Offset from yaw axis to gripper axis & $0 \mathrm{~mm}$ \\
Radius from gripper axis to tip & $17.5 \mathrm{~mm}$ \\
Gripper face length & $14.3 \mathrm{~mm}$ \\
Range of motion & \\
Radius of arc-shape guide & $1.5+(0.45 / 2) \mathrm{mm}$ \\
Rotation radius of wire tip around pitch axis & $9.45+(0.45 / 2) \mathrm{mm}$ \\
Pitch & $\pm 90^{\circ}$ \\
\hline
\end{tabular}

Table 2 List of the components of the wrist mechanism for comparing the proposed and conventional mechanisms

\begin{tabular}{lll}
\hline Part name & $\begin{array}{l}\text { Proposed } \\
\text { mechanism }\end{array}$ & $\begin{array}{l}\text { Conventional } \\
\text { mechanism [7] }\end{array}$ \\
\hline $\begin{array}{ll}\text { Complex shape parts } \\
\text { Supporting part }\end{array}$ & 1 & 1 \\
Pitch part & 1 & 1 \\
Right finger part & 1 & 1 \\
Left finger part & 1 & 1 \\
Simple shape parts & & \\
Supporting shaft & 2 & 3 \\
Pulley & 0 & 8 \\
Stainless wire $(\phi 0.45 \mathrm{~mm}, 7 \times 19)$ & 3 & 3 \\
Total & 9 & 18 \\
\hline
\end{tabular}

very important that surgeons be able to precisely control the grasping force. However, in a conventional wire-andpulley setup that includes an interference mechanism like the da Vinci [5], when the pitch motion is operated manually, the yaw and gripping axes are moved unexpectedly by the interference mechanism. It is difficult to design a manual drove handle that is free from the influence of the interference mechanism, and thus, the handle must become a rather complicated apparatus.

\section{Results and discussion Prototype model}

Based on the abovementioned mechanical design considerations, a prototype model of the proposed wrist mechanism was developed. Manufacturing of the parts and assembly of the mechanism were carried out smoothly. The assembly of wire routing, in particular, was extremely easy. Figures 15 and 16 show an overview and a close-up of the wrist mechanisms of articulated forceps, respectively.

\section{Evaluation experiments Range of motion results}

Figure 17 shows the range of motion of the pitch, yaw, and gripper axis. These data show that the specifications listed in Table 2 have been satisfied without the motion range of pitch angle. The motion range of pitch angle is slightly less than $90^{\circ}$. The reason is the wire pitch angle becomes larger than $90^{\circ}$ since it is necessary to pass two wires of yaw and gripper axis.

\section{Transmission mechanism efficiency and no-load input torque results}

In the test shown in Fig. 18, the input torque $T_{i}$ applied to the drive shaft pulley was measured by a force gauge and the output torque $T_{o}$ was measured by a force sensor. 


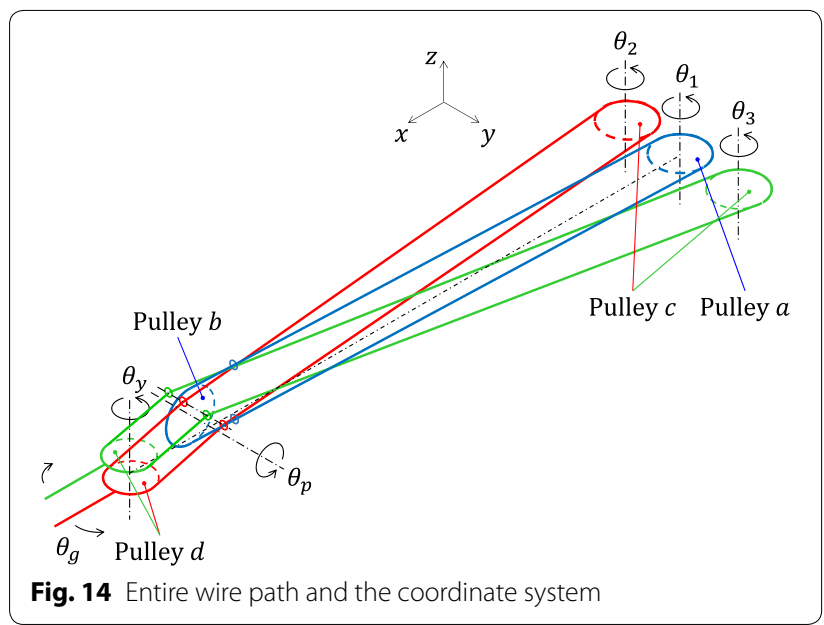

Table 3 Pulley diameter and pitch diameter

\begin{tabular}{lll}
\hline Pulley name & Pulley diameter $(\mathbf{m m})$ & $\begin{array}{l}\text { Pitch } \\
\text { diameter } \\
(\mathbf{m m})\end{array}$ \\
\hline$a$ & $\phi 7$ & $\phi 7.45$ \\
$b$ & $\phi 5$ & $\phi 5.45$ \\
$c$ & $\phi 7$ & $\phi 7.45$ \\
$d$ & $\phi 5$ & $\phi 5.45$ \\
\hline
\end{tabular}

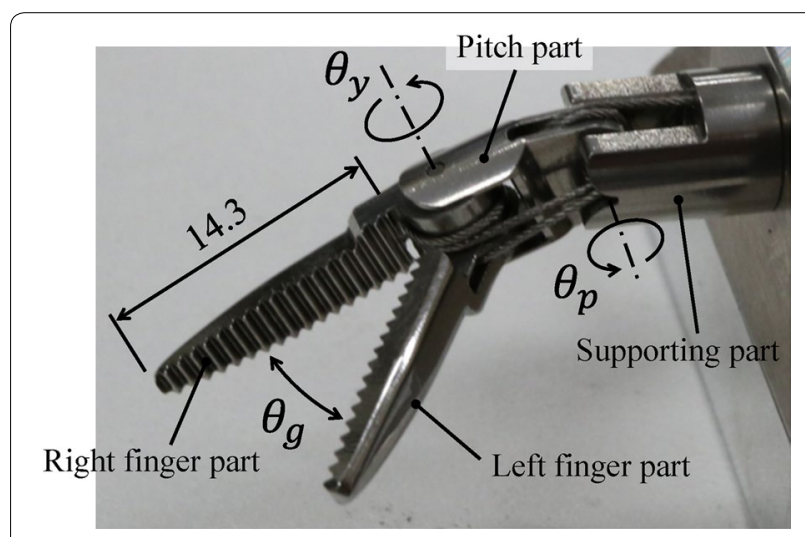

Fig. 15 Overview of the wrist mechanism of articulated forceps

The drive mechanism efficiency $\eta$ was then calculated for each axis using the equation $\eta=\frac{T_{o}}{R T_{i}}$, where $R$ is the reduction ratio (pulley ratio including the wire diameter) of each axis. In this case, $R=\frac{5.45}{7.45}=0.73$ from Table 3 . The input torque was calculated by $F_{i} r_{i}$, and the output torque was calculated by $F_{o} r_{o}$ because the angular error between the force direction and the detection direction is less than $5^{\circ}$.

In the experimental results shown below, the input torque is given by $R F_{i} r_{i}$ to eliminate the influence of the reduction ratio. The maximum pitch axis input torque is approximately $140 \mathrm{mNm}$, and the maximum yaw axis input torque is approximately $100 \mathrm{mNm}$. The input torque is determined by the ratio of the lengths from the pitch axis and the yaw axis to the tip, which is $24.45 \mathrm{~mm}: 17.5 \mathrm{~mm} \fallingdotseq 1.4: 1$.

Figure 19 shows the relation between the input torque $R F_{i} r_{i}$ and the output torque $F_{o} r_{o}$ in the measured data. Figure 19a shows the pitch axis data for a pitch angle of $60^{\circ}$, and Fig. 19b shows the yaw axis data for a pitch angle of $40^{\circ}$. The yaw and gripper angles are both $0^{\circ}$. The noload input torque refers to the input torque at the time when the output torque appears. The remaining output torque refers to the output torque after the input torque has been released. Figure 20 shows the drive mechanism efficiency of the pitch and yaw axes for pitch angles from $0^{\circ}$ to $80^{\circ}$ (yaw angle $=0^{\circ}$ ). The gripper axis is same as the yaw axis. Figure 21 shows the no-load input torque of the pitch and yaw axes for pitch angles from $0^{\circ}$ to $80^{\circ}$ (yaw angle $=0^{\circ}$ ). The remaining output torque ranges from 0 to $25 \mathrm{mNm}$.

Regarding the pitch axis, the efficiency from $0^{\circ}$ to $60^{\circ}$ is more than $80 \%$. However, beyond $60^{\circ}$, the efficiency decreases rapidly, and the no-load torque increases. As shown in Fig. 9, the wire pass length errors of the yaw and gripper axes increase rapidly when the pitch angle exceeds $70^{\circ}$. Therefore, torque is generated around the pitch axis in order to extend the wire of the yaw and gripper axes. As shown in Fig. 17b, because there are two wires (the yaw and gripper wires) through the pitch joint, the pitch joint angle is different from the wire pitch angle. The angle between the two wires is approximately $10^{\circ}$. Therefore, the region of decreasing efficiency (beyond $60^{\circ}$ ) is not equal to the region of increasing wire pass length error (beyond $70^{\circ}$ ).

Contrastingly, in the case of the yaw axis, the efficiency gently decreases, and the no-load torque increases, which are caused by increasing friction between the wire and the arc-shaped guides associated with the wire pass length error and increase in winding angle.

As shown in the above experimental results, the transmission mechanism efficiency and no-load torque of the proposed mechanism are inferior to the wire-and-pulley conventional mechanism like the da Vinci [5]. The proposed mechanism needs to be improved by increasing the transmission mechanism efficiency and decreasing the no-load input torque, or by reducing the pitch motion range.

\section{Demonstrated motion results}

The effect of the reduction in the wire path length error was confirmed by the smooth manual operation of the 1 st axis in the vicinity of $\theta=0^{\circ}$. Furthermore, tests 


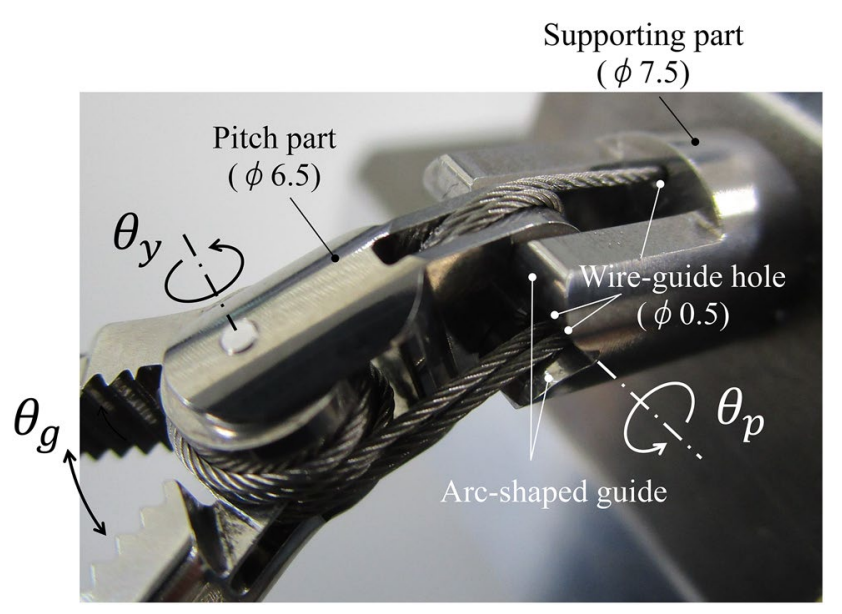

Fig. 16 Close-up of the wrist mechanism of articulated forceps

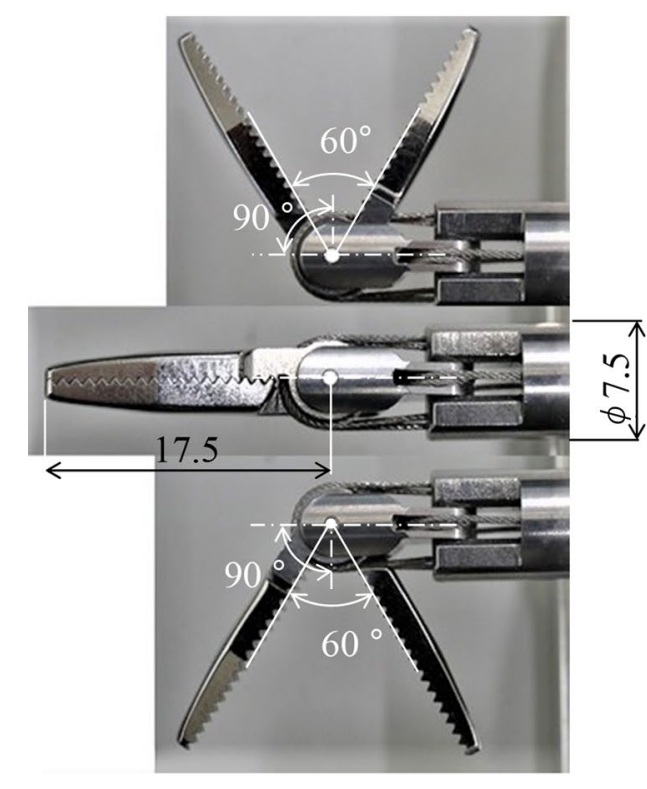

a

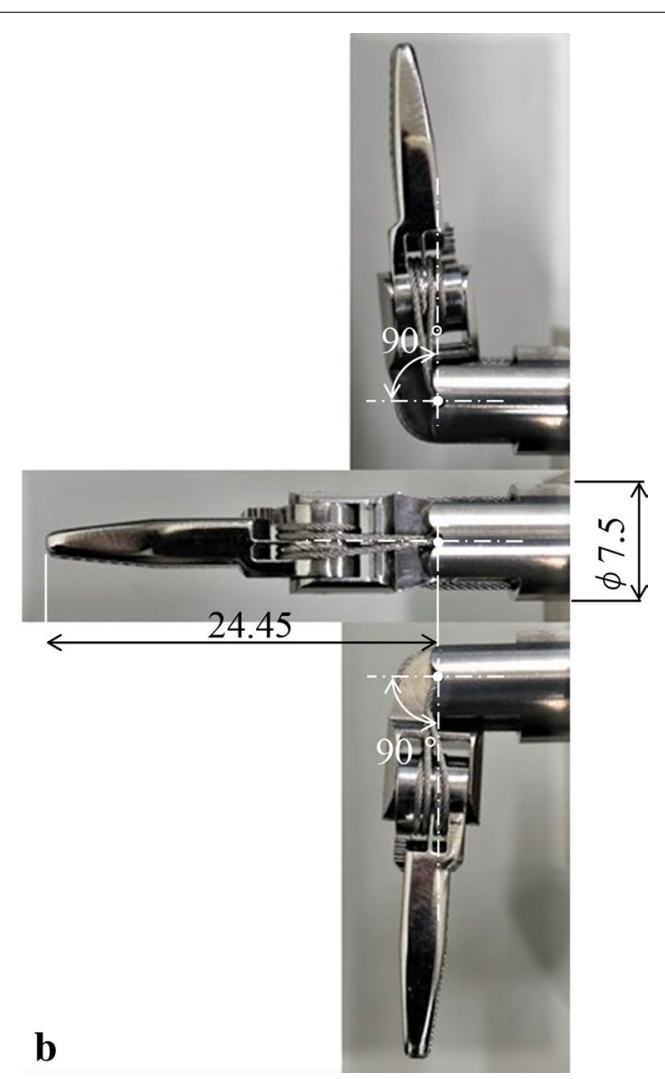

Fig. 17 Motion range. a Yaw- and gripper-axis motion range (top view); b pitch-axis motion range (front view)

demonstrated that the motion of the each of the axes, yaw, pitch, and gripper axes was operated by a command from the tactile-switch user interface. Servo motors HS8057MH (Hitec Multiplex Japan, Inc.) were used for the drive motors, and an Arduino Leonardo microcontroller was used. Figure 22 shows an overview of the test bench.
An additional movie file shows this greater detail (see Additional file 1).

These results confirmed that the proposed mechanism has the fundamental functions and performance characteristics that make it a feasible wrist mechanism of articulated forceps for robot-assisted laparoscopic surgery. 


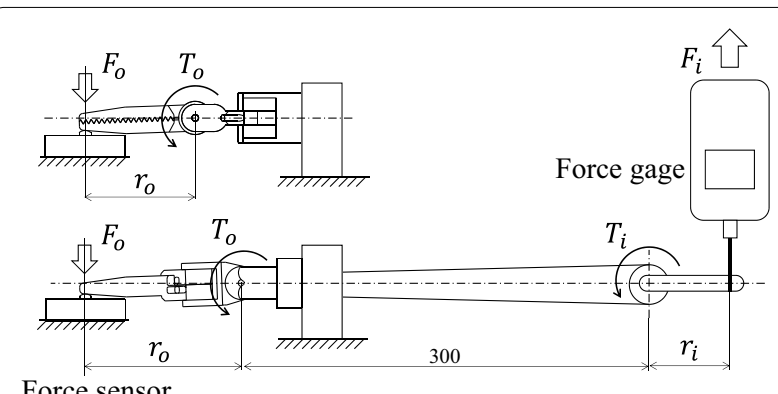

Force sensor

Fig. 18 Measurement of drive mechanism efficiency and no-load input torque

Conversely, it is clear that the disadvantages of the proposed mechanism lie in the transmission mechanism efficiency and no-load input torque.

\section{Conclusions}

Considering the demerits of the interference mechanism regarding the use of articulated forceps in laparoscopic surgery, a new wrist mechanism had been proposed. The kinematic model of this mechanism is decoupled between the pitch axis and yaw axis by a very simple mechanism that employs arc-shaped guides and wire guide holes. The arc-shaped guides minimize the wire path length error of yaw and gripper axis wire due to the motion of the pitch axis. The optimized position of the arc-shaped guides is decided by the minimal RMS of the wire path length error. The proposed wrist mechanism has only half the number of parts as compared to the previously developed clinical use robotic forceps [5, 7]. Furthermore, the effectiveness of the proposed mechanism was demonstrated by using a prototype model with a maximum outer diameter of $7.5 \mathrm{~mm}$. Conversely, the disadvantages of the proposed mechanism lie in the

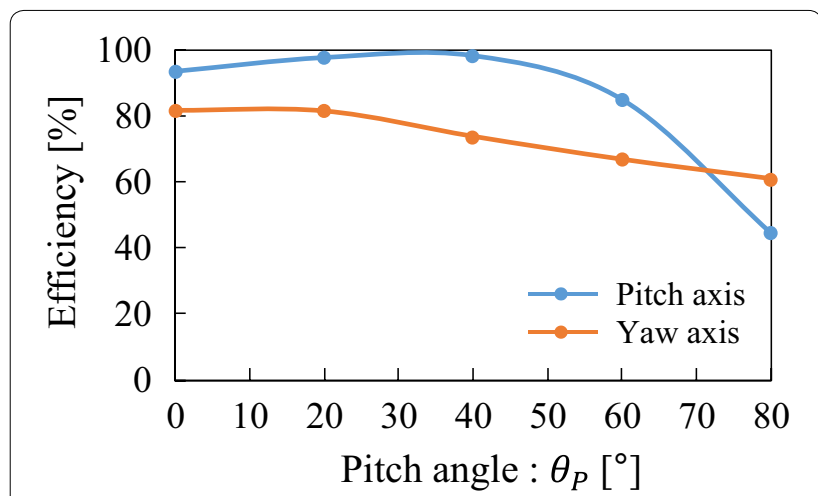

Fig. 20 Drive mechanism efficiency (yaw angle $=0^{\circ}$ ). a Pitch axis; $\mathbf{b}$ yaw axis

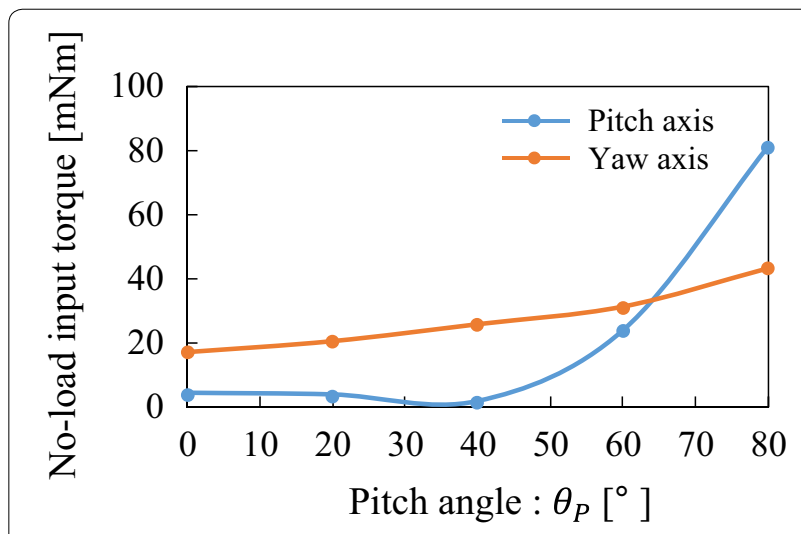

Fig. 21 No-load input torque (yaw angle $=0^{\circ}$ ). a Pitch axis; $\mathbf{b}$ yaw axis

transmission mechanism efficiency and no-load input torque.

Future works toward the final robot system are planned as follows.

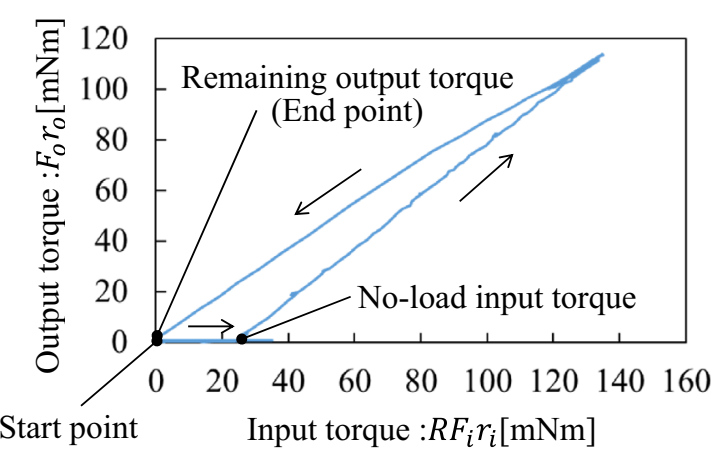

a

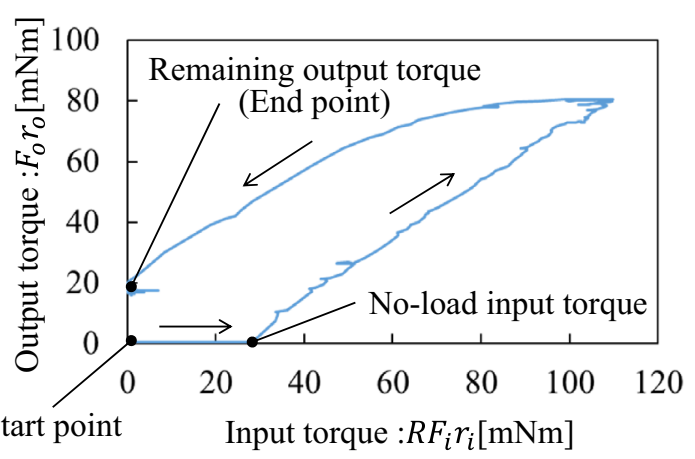

b

Fig. 19 The relation between the input torque and the output torque in the measured data. a Pitch axis (pitch angle $=60^{\circ}$, yaw angle $=0^{\circ}$, gripper angle $=0^{\circ}$ ); $\mathbf{b}$ yaw axis (pitch angle $=40^{\circ}$, yaw angle $=0^{\circ}$, gripper angle $=0^{\circ}$ ) 


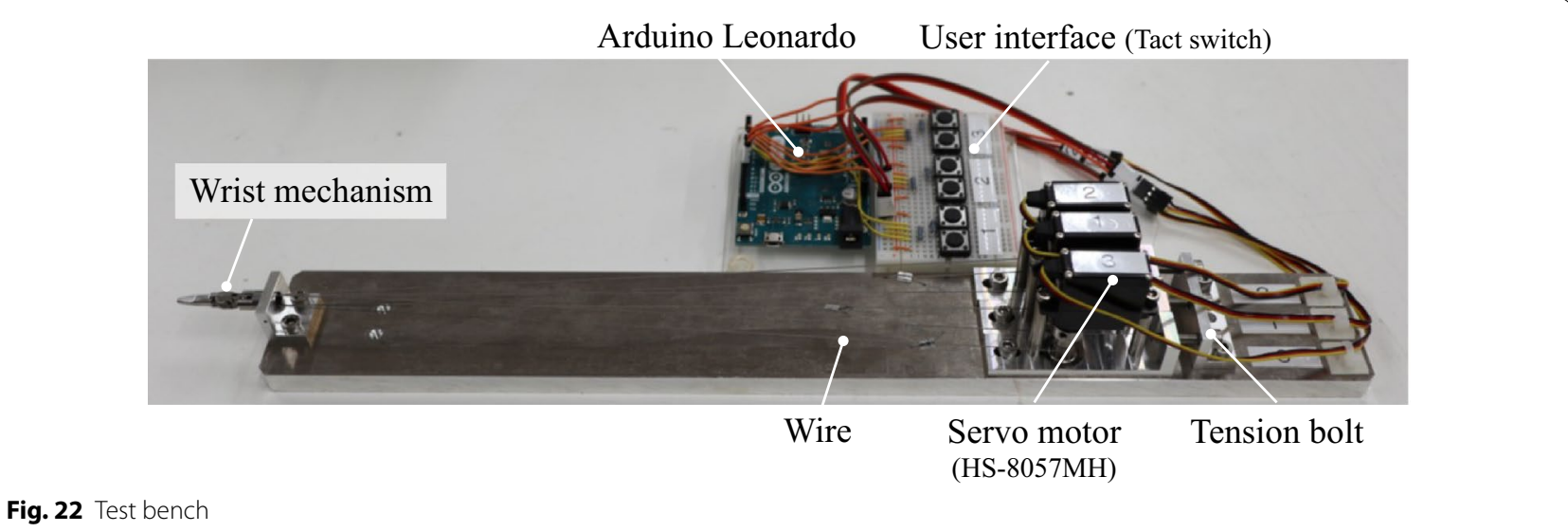

1. Further analysis and improvement of the noninterference drive mechanism, with a particular emphasis on increasing the transmission mechanism efficiency, decreasing the no-load input torque, and improving the durability of the wire around the arc-shaped guides.

2. The implementation of this wrist mechanism to slave manipulators and/or handheld articulated forceps considering the clinical environment.

3. The development of a master-slave manipulator system for laparoscopic surgery for example, using a 6-axis vertical articulated robot [17].

\section{Additional file}

Additional file 1. Demonstration motion of each axis by a tactile-switch user interface.

\section{Authors' contributions}

The author read and approved the final manuscript

\section{Acknowledgements}

Not applicable.

\section{Competing interests}

The author declares that there is no competing interests.

\section{Availability of data and materials \\ Not applicable.}

\section{Ethics approval and consent to participate}

Not applicable.

\section{Funding}

Kokushikan University internal fund.

\section{Publisher's Note}

Springer Nature remains neutral with regard to jurisdictional claims in published maps and institutional affiliations.
Received: 27 December 2018 Accepted: 14 January 2019

Published online: 22 January 2019

\section{References}

1. Intuitive Surgical, Inc., the da Vinci ${ }^{\circledR}$ Surgical System. https://www.intui tive.com/. Accessed 3 Nov 2018

2. FlexDex Inc. FlexDex. https://flexdex.com/. Accessed 3 Nov 2018

3. Tuebingen Scientific Medical GmbH, Radius Surgical System. http://www. tuebingen-scientific.com/. Accessed 1 Feb 2018

4. Hackethal A, Koppan M, Eskef K, Tinneberg H-R (2012) Handheld articulating laparoscopic instruments driven by robotic technology. First clinical experience in gynecological surgery. Gynecolog Surg 9(2):203-206

5. Wallace DT, Julian CA, Morley TA, Baron DS (2002) Surgical tools for use in minimally invasive telesurgical applications. US Patent 6,394,998, 28 May 2002

6. Jinno M, Sunaoshi T, Miyagawa T, Hato T, Matsuhira N, Morikawa Y, Ozawa S, Kitajima M (2006) Development of robotic forceps for laparoscopic surgery. J Robot Mechatron 18(3):249-254

7. Jinno M (2017) Proof of concept for a wrist mechanism for articulated forceps for use in robot assisted laparoscopic surgery. Robomech J 5:5

8. Jinno M, Sunaoshi T, Omori S (2012) Working mechanical device and manipulator. US Patent 7,942,895, 17 May 2011

9. Jinno M (2012) Manipulator. US Patent 8,277,443, 2 Oct 2012

10. Nishizawa K, Kishi K (2004) Development of interference-free wire driven joint mechanism for surgical manipulator systems. J Robot Mechatron 16(2):116-121

11. Braun M (2012) Surgical instrument comprising an instrument handle and zero point adjustment. US Patent 8,267,958, 18 Sept 2012

12. Ikuta K, Yamamoto K, Sasaki K (2003) Development of remote microsurgery robot and new surgical procedure for deep and narrow space. In: Proceedings IEEE international conference on robotics and automation, pp 1103-1108

13. Awtar S, Nielsen J, Trutna T, Mansfield A, Abani R, Geiger J, Quigley P (2014) Minimal access tool. US Patent 8,668,702, 11 Mar 2014

14. Lee W, Chamorro A (2008) Surgical instrument. US Patent 7,338,513, 4 Mar 2008

15. Haraguchi D, Kanno T, Tadano K, Kawashima K (2015) A pneumaticallydriven surgical manipulator with a flexible distal joint capable of force sensing. IEEE/ASME Trans Mechatron 20(6):2950-2961

16. Jinno M, Sunaoshi T, Omori S (2012) Manipulator and control method therefor. US Patent 8,237,388, 7 Aug. 2012

17. Jinno M (2014) Master-slave manipulator for laparoscopic surgery using a 6-axis vertical articulated robot. Robomech J 1:23 